Mon. Not. R. Astron. Soc. 000,1-?? (?) $\quad$ Printed 17 August $2017 \quad$ (MN LATEX style file v2.2)

\title{
Evidence for a radiatively driven disc-wind in PDS 456?
}

\author{
G. A. Matzeu, ${ }^{1,2 \star}$ J. N. Reeves, ${ }^{2,3}$ V. Braito, ${ }^{1}$ E. Nardini, ${ }^{4}$ D. E. McLaughlin, ${ }^{2}$ \\ A. P. Lobban, ${ }^{2}$ F. Tombesi ${ }^{5,6,7}$ and M. T. Costa ${ }^{2}$ \\ ${ }^{1}$ INAF - Osservatorio Astronomico di Brera, Via Bianchi 46, I-23807 Merate (LC), Italy \\ ${ }^{2}$ Astrophysics Group, School of Physical and Geographical Sciences, Keele University, Keele, Staffordshire ST5 5BG, UK \\ ${ }^{3}$ Center for Space Science and Technology, University of Maryland Baltimore County, 1000 Hilltop Circle, Baltimore, MD 21250, USA \\ ${ }^{4}$ INAF - Osservatorio Astrofisico di Arcetri, Largo Enrico Fermi 5, I-50125 Firenze, Italy \\ ${ }^{5}$ X-ray Astrophysics Laboratory, NASA/Goddard Space Flight Center, Greenbelt, MD, 20771, USA \\ ${ }^{6}$ Department of Astronomy and CRESST, University of Maryland, College Park, MD, 20742, USA \\ ${ }^{7}$ Department of Physics, University of Rome "Tor Vergata", Via della Ricerca Scientifica 1, I-00133 Rome, Italy
}

17 August 2017

\begin{abstract}
We present a newly discovered correlation between the wind outflow velocity and the X-ray luminosity in the luminous $\left(L_{\mathrm{bol}} \sim 10^{47} \mathrm{erg} \mathrm{s}^{-1}\right)$ nearby $(z=0.184)$ quasar PDS 456 . All the contemporary XMM-Newton, NuSTAR and Suzaku observations from 2001-2014 were revisited and we find that the centroid energy of the blueshifted $\mathrm{Fe} \mathrm{K}$ absorption profile increases with luminosity. This translates into a correlation between the wind outflow velocity and the hard X-ray luminosity (between 7-30 keV) where we find that $v_{\mathrm{w}} / c \propto L_{7-30}^{\gamma}$ where $\gamma=0.22 \pm 0.04$. We also show that this is consistent with a wind that is predominately radiatively driven, possibly resulting from the high Eddington ratio of PDS 456.
\end{abstract}

Key words: Subject headings: Black hole physics - galaxies: active - galaxies: nuclei quasars: individual (PDS 456) - X-rays: galaxies

\section{INTRODUCTION}

Blueshifted Fe $\mathrm{K}$ shell absorption lines, observed at $>7 \mathrm{keV}$ in the rest-frame X-ray spectra of AGN, were first discovered in luminous quasars (e.g., Chartas, Brandt \& Gallagher 2003, Reeves, O’Brien \& Ward 2003; Pounds et al. 2003). The high velocities inferred, i.e. $v_{\mathrm{w}} \gtrsim 0.1 c$, imply an association with an accretion disc-wind. The importance of these winds is supported by their frequent detection, as they are observed in the X-ray spectra of approximately $40 \%$ of AGN (Tombesi et al. 2010, Gofford et al. 2013), suggesting that their geometry is characterized by a wide opening angle as recently confirmed in the luminous quasar PDS 456 by Nardini et al. (2015, hereafter N15). These outflows are characterized by considerably high column densities $\left(N_{\mathrm{H}} \sim 10^{23} \mathrm{~cm}^{-2}\right)$ and a mean velocity $\left\langle v_{\mathrm{w}}\right\rangle \sim 0.1 c$ (Tombesi et al. 2010) up to mildly relativistic values of $\sim 0.2-0.4 c$ in the most extreme cases (e.g., Chartas et al. 2002, Reeves et al. 2009, Tombesi et al. 2015). The high velocities and high columns can result in a large amount of mechanical power, possibly exceeding the $0.5-5 \%$ of the bolometric luminosity ( $L$ hereafter), as required for significant AGN feedback King 2003, King \& Pounds 2003, Di Matteo, Springel \& Hernquist 2005, Hopkins \& Elvis 2010). In principle the physical properties of these fast winds can provide more insight on the mechanism through which they are driven/accelerated. The possible sce-

^ Correspondence to: gabriele.matzeu@ brera.inaf.it narios are, radiatively driven winds (e.g., Proga, Stone \& Kallman 2000, Proga \& Kallman 2004) and/or magneto-centrifugally driven winds (MHD wind hereafter, e.g. Ohsuga et al. 2009, Kazanas et al. 2012). Most outflow studies so far mainly concentrated on radiatively driven winds (e.g., Sim et al. 2010, Reeves et al. 2014, Hagino et al. 2015, Nomura \& Ohsuga 2017). However, recently Fukumura et al. (2017) successfully applied an MHD wind model to the microquasar GRO J1655-40 suggesting that a similar mechanism applies across the black hole mass and luminosity scales.

The quasar PDS 456, located at redshift $z=0.184$ (Torres et al. 1997) is the most luminous ( $L \sim 10^{47} \mathrm{erg} \mathrm{s}^{-1}$; Simpson et al. 1999. Reeves et al. 2000) radio-quiet quasar in the local Universe $(z<0.3)$. Since the first XMM-Newton (40 ks) observation carried out in 2001 (Reeves, O'Brien \& Ward 2003), PDS 456 has showed the presence of a persistent deep absorption trough in the Fe K band above $7 \mathrm{keV}$. This can be associated to highly ionized Fe K-shell absorption with a corresponding outflow velocity of $v_{\mathrm{w}} \sim 0.25-$ $0.3 c$ (Reeves et al. 2009, 2014, Gofford et al. 2014, N15; Matzeu et al.2016 hereafter M16), which originates from an accretion disk wind. In this letter we present a new correlation between the discwind velocity derived from the Fe $\mathrm{K}$ profile and the intrinsic X-ray luminosity, which we interpret in the framework of a radiatively driven wind. Values of $H_{0}=70 \mathrm{~km} \mathrm{~s}^{-1} \mathrm{Mpc}^{-1}$ and $\Omega_{\Lambda_{0}}=0.73$ are assumed throughout this work and errors are quoted at the $1 \sigma$ confidence level or $90 \%$ where otherwise stated.

(c) ? RAS 


\begin{tabular}{ccccc}
\hline Obs. & Satellite & Start Date (UT) & $\begin{array}{c}\text { Exposure } \\
(\mathrm{ks})\end{array}$ & Ref. \\
\hline 2001 & XMM-Newton & $2001-02-2609: 44$ & 44.0 & $(1)$ \\
2007 & XMM-Newton & $2007-09-1201: 07$ & 179.0 & $(2)$ \\
2007 & Suzaku & $2007-02-24,17: 58$ & 190.6 & $(3)$ \\
2011 & Suzaku & $2011-03-16,15: 00$ & 125.6 & $(4)$ \\
$2013 \mathrm{a}$ & Suzaku & $2013-02-21,21: 22$ & 182.3 & \\
$2013 \mathrm{~b}$ & Suzaku & $2013-03-03,19: 43$ & 164.8 & $(5,6)$ \\
$2013 \mathrm{c}$ & Suzaku & $2013-03-08,12: 00$ & 108.3 & \\
$\mathrm{~A}$ & XMM-Newton & $2013-08-27,04: 41$ & 95.7 & \\
& NuSTAR & $2013-08-27,03: 41$ & 43.8 & \\
$\mathrm{~B}$ & XMM-Newton & $2013-09-06,03: 24$ & 95.7 & \\
& NuSTAR & $2013-09-06,02: 56$ & 43.0 & \\
$\mathrm{C}$ & XMM-Newton & $2013-09-15,18: 47$ & 102.0 & $(7)$ \\
& NuSTAR & $2013-09-15,17: 56$ & 44.0 & \\
$\mathrm{D}$ & XMM-Newton & $2013-09-20,02: 47$ & 93.0 & \\
& NuSTAR & $2013-09-20,03: 06$ & 58.6 & \\
$\mathrm{E}$ & XMM-Newton & $2014-02-26,08: 03$ & 100.3 & \\
& NuSTAR & $2014-02-26,08: 16$ & 109.5 & \\
\hline
\end{tabular}

Table 1. Summary of the Suzaku, XMM-Newton and NuSTAR observations of PDS 456 performed from 2001-2014. The net exposure for NuSTAR are for a single FPMA/B detector while XMM-Newton and Suzaku net exposures are based on the EPIC-pn and individual XIS CCD detectors respectively. References: (1) Reeves, O'Brien \& Ward (2003), (2) Behar et al. (2010), (3) Reeves et al. (2009), (4) Reeves et al. (2014), (5) Gofford et al. (2014), (6) M16, (7) N15.

\section{WIND ANALYSIS}

We utilize all the available XMM-Newton, Suzaku and NuSTAR datasets, concentrating on the X-ray band above $2 \mathrm{keV}$ fitted with a simple power-law continuum modified by Galactic absorption $\left(N_{\mathrm{H}}=2.0 \times 10^{21} \mathrm{~cm}^{-2}\right)$ and a single layer of neutral partial covering absorption to account for any spectral curvature with velocity set to be equal to the fast wind's outflow velocity (see M16). A broad $\mathrm{Fe} \mathrm{K}$ emission line at $7 \mathrm{keV}$ was also included, where the width was fixed to that measured by the absorption line when the latter is modelled with a simple Gaussian. The X-ray spectra were adopted from previous work (see Table 1 for details). In Fig.1 the residuals of the $\mathrm{Fe} \mathrm{K}$ absorption profiles as a ratio to a power-law continuum are plotted for four of the twelve observations with different X-ray luminosities (in decreasing order). It is evident that the centroid energy increases across the different epochs with increasing luminosity.

As an initial test we investigate how the energy of the Fe K profile responds to the ionizing luminosity, measured in either the $7-30 \mathrm{keV}$ band ( $L_{7-30}$ hereafter) or the $2-10 \mathrm{keV}$ band $\left(L_{2-10}\right)$ between all the sequences. The $7-30 \mathrm{keV}$ band was chosen as it is above the Fe $\mathrm{K}$ absorption edge threshold and is where the primary continuum emerges. This luminosity is absorption corrected and is either inferred directly from the NUSTAR data or from extrapolating the best fit model above $10 \mathrm{keV}$, for those sequences where no NuSTAR observation is available $\square^{1}$ Monte Carlo simulations were performed in order to estimate the uncertainties on the absorptioncorrected intrinsic luminosity. For each observation, 1000 simulations were performed with the XSPEC command fakeit, assuming the best-fit models obtained from the spectral analysis of the actual data. Each simulated spectrum was fitted again to take into account the uncertainties on the null hypothesis, allowing the main

1 The extrapolation can be considered a good assumption, given that all the NuSTAR observations of PDS 456 indicate a continuum shape of a simple power-law $(\Gamma=2.2-2.6)$ without any strong hard excess.

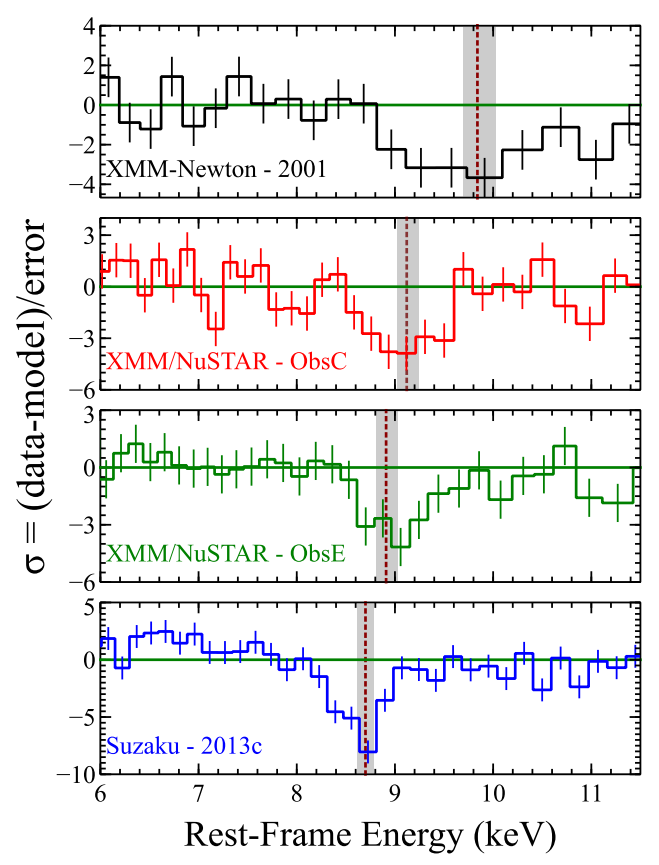

Figure 1. Residuals to the power-law continuum fitted between $2-10 \mathrm{keV}$ band from four selected observations of PDS 456 decreasing in X-ray luminosity. This shows how the centroid energy of the Fe K absorption profile generally decreases in energy with decreasing luminosity from top to bottom. The vertical dotted lines and the gray shadowed areas indicate the position of the centroid and the $90 \%$ uncertainty (see Table 2).

spectral parameters vary. These are the photon index and normalization of the continuum, the covering fraction and column density of the partial covering absorber, as well as the column and outflow velocity of the ionized absorber. With the new model, a second simulation was run and fitted, from which we derived the distribution of the luminosities, which were well approximated by a Gaussian distribution. The subsequent $1 \sigma$ uncertainty was used as the input to the correlation analysis. We also extracted the simulated distributions of the main spectral parameters to check that they were representative of the uncertainties of the actual best fit models. Thus the derived errors on the luminosities fully account for the uncertainties on each of the free spectral parameters.

We first parametrize the blueshifted absorption lines with a Gaussian profile, whose main parameters are tabulated in Table 2 In Fig. 2 we show the centroid energy versus $L_{7-30}$ which becomes more blueshifted at higher luminosities suggesting a positive correlation with the intrinsic luminosity. Thus from the centroid energy we can infer the corresponding outflow velocity $\left(v_{\mathrm{w}} / c\right)$ of the material, assuming it is mainly associated with FeXXVI Ly $\alpha$ rather than Fe XXV He $\alpha$ (see Table 2). The top panel of Fig. 3 shows the outflow velocity derived from the Gaussian fit against $L_{7-30}$ plotted in $\log -\log$ space. Subsequently the data points were fitted with a standard regression line of the form $\log \left(v_{\mathrm{w}} / c\right)=m \log \left(L_{7-30}\right)+$ $\log (C)$, performed through the bivariate correlated errors and intrinsic scatter algorithm (BCES, Akritas \& Bershady 1996) which is one of the most common methods that takes into account errors in both $\mathrm{x}$ and $\mathrm{y}$ values. The regression line produced a gradient of $m=0.20 \pm 0.05$ where the shaded magenta area indicates the slope dispersion (at $1 \sigma$ confidence level). A comparable regression fit is found with the intrinsic $2-10 \mathrm{keV}$ luminosity $\left(L_{2-10}\right)$, with $m=0.22 \pm 0.04$ (see Table 3). 


\begin{tabular}{|c|c|c|c|c|c|c|c|}
\hline \multirow[b]{2}{*}{ Satellite } & \multirow[b]{2}{*}{ Obs. } & \multicolumn{3}{|c|}{$\longrightarrow$ Gaussian $\longrightarrow$} & \multicolumn{2}{|l|}{ XSTAR } & \multirow[b]{2}{*}{$\begin{array}{c}L_{7-30 \mathrm{keV}}(6) \\
\left(\times 10^{44} \mathrm{erg} \mathrm{s}^{-1}\right)\end{array}$} \\
\hline & & $\begin{array}{c}\text { E rest }_{\text {ret }}(1) \\
(\mathrm{keV})\end{array}$ & $v_{\mathrm{w}} / c(2)$ & $\begin{array}{c}\mathrm{EW}(3) \\
(\mathrm{eV})\end{array}$ & $v_{\mathrm{w}} / c(4)$ & $\begin{array}{c}L_{2-10 \mathrm{keV}}(5) \\
\left(\times 10^{44} \mathrm{erg} \mathrm{s}^{-1}\right)\end{array}$ & \\
\hline \multirow{5}{*}{ XMM-Newton \& NuSTAR } & A & $9.25_{-0.11}^{+0.18}$ & $0.286_{-0.010}^{+0.017}$ & $-88 \pm 47$ & $0.299_{-0.012}^{+0.012}$ & $7.94 \pm 0.26$ & $5.22 \pm 0.11$ \\
\hline & B & $9.06_{-0.20}^{+0.19}$ & $0.268_{-0.020}^{+0.019}$ & $-295 \pm 63$ & $0.278_{-0.020}^{+0.016}$ & $3.57 \pm 0.31$ & $1.68 \pm 0.06$ \\
\hline & $\mathrm{C}$ & $9.12_{-0.09}^{+0.11}$ & $0.275_{-0.009}^{+0.011}$ & $-359 \pm 55$ & $0.286_{-0.007}^{+0.006}$ & $4.51 \pm 0.26$ & $2.60 \pm 0.08$ \\
\hline & $\mathrm{D}$ & $9.16_{-0.12}^{+0.12}$ & $0.277_{-0.012}^{+0.012}$ & $-256 \pm 60$ & $0.292_{-0.007}^{+0.008}$ & $5.10 \pm 0.31$ & $2.93 \pm 0.08$ \\
\hline & $\mathrm{E}$ & $8.91_{-0.11}^{+0.11}$ & $0.268_{-0.020}^{+0.019}$ & $-405 \pm 60$ & $0.269_{-0.009}^{+0.008}$ & $3.68 \pm 0.35$ & $1.81 \pm 0.06$ \\
\hline \multirow{5}{*}{ Suzaku } & 2007 & $9.26_{-0.13}^{+0.15}$ & $0.287_{-0.013}^{+0.015}$ & $-235 \pm 74$ & $0.296_{-0.012}^{+0.012}$ & $4.35 \pm 0.34$ & $2.32 \pm 0.12$ \\
\hline & 2011 & $9.07_{-0.20}^{+0.18}$ & $0.270_{-0.020}^{+0.018}$ & $-460 \pm 151$ & $0.266_{-0.011}^{+0.011}$ & $4.14 \pm 0.53$ & $2.21 \pm 0.19$ \\
\hline & $2013 \mathrm{a}$ & $8.85_{-0.29}^{+0.33}$ & $0.248_{-0.030}^{+0.035}$ & $>-214$ & $0.248_{-0.020}^{+0.030}$ & $3.53 \pm 0.35$ & $1.88 \pm 0.14$ \\
\hline & $2013 b$ & $\begin{array}{l}8.86_{-0.09}^{+0.09} \\
\end{array}$ & $0.249_{-0.010}^{+0.010}$ & $-390 \pm 81$ & $0.255_{-0.010}^{+0.010}$ & $2.90 \pm 0.36$ & $1.55 \pm 0.16$ \\
\hline & $2013 c$ & $8.70_{-0.08}^{+0.07}$ & $0.234_{-0.009}^{+0.008}$ & $-568 \pm 82$ & $0.240_{-0.010}^{+0.010}$ & $2.77 \pm 0.38$ & $1.46 \pm 0.17$ \\
\hline \multirow{2}{*}{ XMM-Newton } & 2001 & $9.84_{-0.16}^{+0.19}$ & $0.335_{-0.019}^{+0.018}$ & $-337 \pm 94$ & $0.340_{-0.015}^{+0.018}$ & $10.5 \pm 0.07$ & $4.97 \pm 0.28$ \\
\hline & 2007 & $9.51_{-0.14}^{+0.14}$ & $0.309_{-0.013}^{+0.013}$ & $>-71$ & $0.299_{-0.034}^{+0.026}$ & $5.55 \pm 0.29$ & $3.67 \pm 0.14$ \\
\hline
\end{tabular}

Table 2. Summary of the observations and the corresponding properties of the Fe K absorption features. These are obtained by fitting with a Gaussian profile (columns 1-3) and then subsequently with XSTAR (columns 4-6). From the former we measured with the respective 90\% errors on: (1) the rest-frame energy, (2) the inferred outflow velocity and (3) the equivalent width of each line. By modelling with XSTAR we measured the outflow velocity (4), while from the fits, the column density ranges from $\log \left(N_{\mathrm{H}} / \mathrm{cm}^{-2}\right)=23.16_{-0.23}^{+0.28}-24.06_{-0.21}^{+0.18}$ and ionization ranges from $\log \left(\xi / \mathrm{erg} \mathrm{cm} \mathrm{s}{ }^{-1}\right)=5.1_{-0.21}^{+0.11}-6.33_{-0.19}^{+0.08}$. In columns (5) and (6) we report the intrinsic luminosities and their corresponding $1 \sigma$ errors in the $2-10 \mathrm{keV}$ and $7-30 \mathrm{keV}$ bands, respectively.

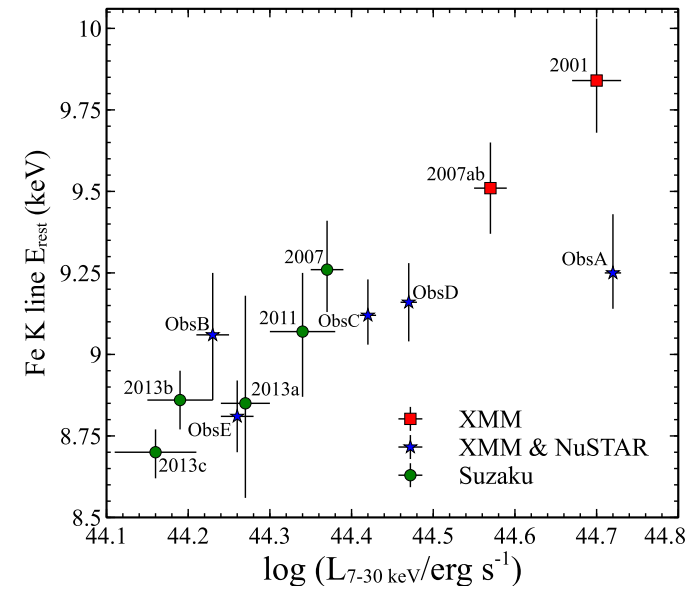

Figure 2. Plot showing how the centroid energy of the Fe K features correlates with $L_{7-30}$ in PDS 456. The distribution of the data favours a positive linear correlation between the two quantities. Suzaku (green circles), XMMNewton \& NuSTAR (blue stars) and XMM-Newton (red squares).

The Fe $\mathrm{K}$ absorption features were then modelled with a more physically motivated self-consistent XSTAR grid, previously adopted in N15 and M16, generated using the UV to hard X-ray SED of PDS 456. The XSTAR model allows the column and/or the ionization to adjust between observations, as well as the net outflow velocity. The corresponding $v_{\mathrm{w}} / c$ values of all twelve observations derived from the XSTAR model are listed in Table 2 and plotted against $L_{7-30}$ on the bottom panel in Fig. 3 The linear regression fit gives a gradient of $m=0.22 \pm 0.04$ consistent with the Gaussian analysis. Similarly, the correlation with $L_{2-10}$ also gives a consistent result of $m=0.24 \pm 0.03$. From both the correlations shown in Fig. 3 it is clear that the outflow velocity of the highly ionized

\begin{tabular}{cccccc}
\hline$x$ & $y$ & $m$ & $\Delta m$ & $\chi_{\nu}^{2}$ & $P_{\text {null }}$ \\
\hline \multicolumn{5}{c}{ From Gaussian } \\
$\log \left(L_{2-10}\right)$ & $\log \left(v_{\mathrm{w}} / c\right)$ & 0.22 & 0.04 & 0.84 & $6.70 \times 10^{-7}$ \\
$\log \left(L_{7-30}\right)$ & 0.20 & 0.05 & 1.08 & \\
\multicolumn{5}{c}{ From XSTAR } \\
$\operatorname{co}\left(L_{2-10}\right)$ & $\log \left(v_{\mathrm{w}} / c\right)$ & 0.24 & 0.03 & 0.82 & $8.29 \times 10^{-8}$ \\
$\log \left(L_{7-30}\right)$ & 0.22 & 0.04 & 1.01 & \\
& & & &
\end{tabular}

Table 3. Results of the linear regressions between the measured outflow velocities (measured from both Gaussian profile and XSTAR grids) and the intrinsic luminosities. $m$ and $\Delta m$ are the gradient and the respective $1 \sigma$ errors respectively, whereas $\chi_{\nu}^{2}$ is the reduced $\chi^{2} /$ dof. $P_{\text {null }}$ is the null hypothesis probability that there is no linear correlation.

material is indeed responding to the intrinsic luminosity, where the most luminous epochs of PDS 456 are characterized by a faster outflow (e.g., $v_{\mathrm{w}} / c=0.296 \pm 0.012$ in the 2007 Suzaku observation and $v_{\mathrm{w}} / c=0.340_{-0.015}^{+0.018}$ in the $2001 X M M$-Newton observation). On the other hand, the low-luminosity 2013 Suzaku observations (i.e. $2013 \mathrm{~b}$ or $2013 \mathrm{c}$, see Tables 1 and 2 present overall the slowest outflows with $v_{\mathrm{w}} / c=0.255 \pm 0.010$ and $v_{\mathrm{w}} / c=0.240 \pm 0.010$, respectively. Thus an important question is: how the changes in luminosity have a direct impact on the outflow velocity in the discwind observed in PDS 456?

\section{DISCUSSION}

\subsection{A radiatively driven wind?}

The positive correlation between the outflow velocity and the ionizing luminosity of PDS 456, may imply that the wind is radiatively driven. The high ionization derived from the wind seen at Fe K in PDS 456, with ionization parameters typically of 

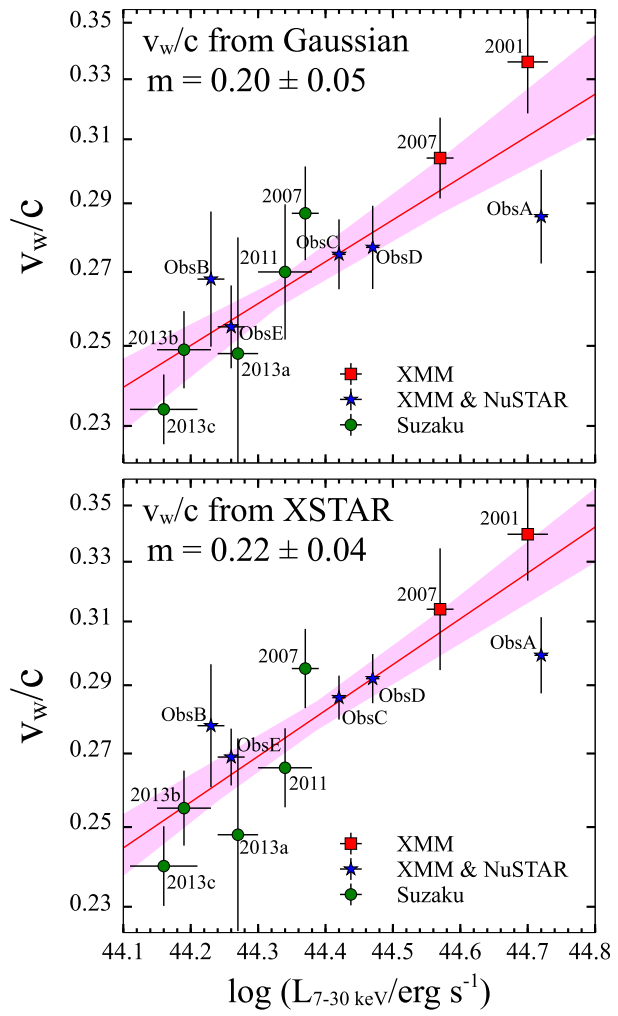

Figure 3. Top: Log-log plot showing the correlation between the outflow velocity, derived from fitting the Fe $\mathrm{K}$ features with a Gaussian profile and the intrinsic $7-30 \mathrm{keV}$ continuum luminosity $\left(L_{7-30}\right)$. The red line shows the linear regression fitted to the data with $1 \sigma$ uncertainty shaded. Bottom: the outflow velocity is now measured by fitting the Fe $\mathrm{K}$ profile with an XSTAR grid where the linear regression produced a gradient of $0.22 \pm 0.04$. These positive correlations suggest that when the source is more luminous the disc-wind is faster.

$\log \left(\xi / \mathrm{erg} \mathrm{cm} \mathrm{s}^{-1}\right) \sim 5-6$ (e.g., N15), might result in a small line opacity, due to only Fe XXV-XXVI. While at first sight this suggests that the dominant interaction mechanism within the photon field is through Thompson scattering (e.g., King \& Pounds 2003), the opacity may be further boosted by the presence of lower ionization X-ray absorption within the wind. This lower ionization gas towards PDS 456 has been observed in the form of the clumpy X-ray partial covering absorber with size scales of $\sim 10 \mathrm{~s}$ of $R_{\mathrm{g}}$ from its variability (M16), as well as through the presence of broad absorption profiles revealed in the soft X-ray band (Reeves et al. 2016). Furthermore blueshifted UV emission and absorption lines from the wind are also present in PDS 456 (O'Brien et al. 2005), similar to those in BAL quasars. Such gas would boost the radiation force acting on the wind, via line driving (see Hagino et al. 2016), resulting in an increase in the force multiplier factor.

To quantify further, we start from the definition of the net force (i.e., radiative minus gravitational force) acting on an electron-ion pair within the wind:

$$
F_{\text {net }}=\mu m_{\mathrm{p}} v\left(r_{\mathrm{w}}\right) \frac{\mathrm{d} v}{\mathrm{~d} r_{\mathrm{w}}}=\frac{L \sigma}{4 \pi r_{\mathrm{w}}^{2} c}-\frac{G M_{\mathrm{BH}} \mu m_{\mathrm{p}}}{r_{\mathrm{w}}^{2}},
$$

where $\mu=n_{\mathrm{H}} / n_{\mathrm{e}} \sim 1.2$ is a constant factor accounting for cosmic elemental abundances, $m_{\mathrm{p}}$ is the proton mass, $r_{\mathrm{w}}$ is the radial distance from the central X-ray source and $\sigma=\sigma_{\mathrm{PE}}+\sigma_{\mathrm{T}}$ is the total cross-section (photoelectric plus Thomson). Rearranging equation (1), gives the equation of motion within the wind:

$$
v\left(r_{\mathrm{w}}\right) \mathrm{d} v=\left(\frac{L \sigma}{4 \pi r_{\mathrm{w}}^{2} \mu m_{\mathrm{p}} c}-\frac{G M_{\mathrm{BH}}}{r_{\mathrm{w}}^{2}}\right) \mathrm{d} r_{\mathrm{w}} .
$$

Now we define $r_{\mathrm{w}}=R_{\text {in }}$ as the launch radius and $v=v_{i}$ as the initial velocity of the disc-wind. At larger radii we assume that $r_{\mathrm{w}} \gg R_{\text {in }}$ and hence $r_{\mathrm{w}} \rightarrow \infty$ and $v \rightarrow v_{\mathrm{w}}$ (i.e., the terminal speed at large radii). Integrating equation (2) between these limits and rearranging gives:-

$$
v_{\mathrm{w}}^{2}-v_{i}^{2}=\frac{2 G M_{\mathrm{BH}}}{R_{\mathrm{in}}}\left(\frac{L \sigma}{4 \pi G M_{\mathrm{BH}} \mu m_{\mathrm{p}} c}-1\right) .
$$

On the right hand side, the first term is the escape velocity of the particle and the second term is essentially the local $L / L_{\text {Edd }}$ within the wind, where the condition $\sigma>\sigma_{\mathrm{T}}$ results from the possible contribution of line driving within the wind, giving a force multiplier factor $>1$. Thus if we simplify the above equation such that $v_{\mathrm{w}}^{2} \gg v_{i}^{2}$ and that locally $L>L_{\mathrm{Edd}}$ (e.g., as a result of the force multiplier), we get a dependence between the outflow velocity, the luminosity and $R_{\text {in }}$ :

$\frac{v_{\mathrm{w}}}{c} \sim\left(\frac{\sigma}{2 \pi \mu m_{\mathrm{p}} c^{3}}\right)^{1 / 2} k_{7-30}^{1 / 2} L_{7-30}^{1 / 2} R_{\mathrm{in}}^{-1 / 2} \propto k_{7-30}^{1 / 2} L_{7-30}^{1 / 2} R_{\mathrm{in}}^{-1 / 2}$.

Here $L=k_{7-30} L_{7-30}$, where $k_{7-30}$ is the bolometric correction factor ( $\gtrsim 100$ for PDS 456).

Our relationship expressed in equation (4) between the outflow velocity and the ionizing luminosity is 'steeper' than what is observed in Fig. 3 There are two possibilities that may explain this; firstly the wind launch radius, $R_{\text {in }}$, may not be constant with luminosity, or $k_{7-30}$ is variable. In the first scenario as the luminosity increases, the innermost parts of the wind can become fully ionized. Subsequently at higher luminosities the innermost fastest streamlines of the wind become effectively unobservable. Given that the ionization parameter varies as $\xi \propto L r_{\mathrm{w}}^{-2}$, the effective radius at which the wind is observable increases with luminosity, leading to the 'flattening' of the slope as observed.

In the latter case the terminal velocity should be proportional to the whole bolometric luminosity rather than just what is directly measured in the hard X-ray band. Furthermore, all of the XMMNewton observations of PDS 456 clearly show that the UV flux is considerably less variable than in the X-ray band (see Fig. 2 Matzeu et al. 2017). As a result $k_{7-30}$ might be variable as a function of luminosity which again may 'flatten' the expected correlation. While it is not currently possible to quantitatively test the relation between $v_{\mathrm{w}} / c$ and $L$ (as only the XMM-Newton observations have simultaneous optical/UV photometry), the resulting correlation between $v_{\mathrm{w}} / c$ and $L$ is at least qualitatively consistent with a steeper slope of $\sim 0.5$. Ongoing optical/UV and X-ray monitoring of PDS 456 with Swift will allow us to measure the bolometric correction as a function of luminosity

One final question is whether it is plausible to radiatively accelerate the wind up to $0.3 c$ ? From equation (3) and taking $L \sim$ $L_{\mathrm{Edd}}$ for PDS 456 , then for a reasonable launch radius of $30 R_{\mathrm{g}}$ (given the rapid wind variability, (M16), then only a modest force multiplier factor of $\tau=\sigma / \sigma_{\mathrm{T}}=2.5$ is required. Furthermore, if PDS 456 is mildly super-Eddington (e.g. $2 L_{\text {Edd }}$ ), then $\tau$ approaches unity. Thus the observed velocities can be easily reproduced within this framework. Note that a comparable positive cor- 
relation between the outflow velocity and luminosity has been recently found by Gofford et al. (2015) and Fiore et al. (2017), the latter from collating a sample of known AGN outflows.

\subsection{MHD launching scenario}

Alternatively, could MHD winds reproduce the above correlation? While to date there is no expected correlation in the MHD wind scenarios (e.g., Ohsuga et al. 2009, Kazanas et al. 2012), the observed velocity can still depend on the continuum photon index, the SED shape and in particular the UV to X-ray slope, $\alpha_{\text {ox }}$.

Indeed Fukumura et al. (2010) predicted that sources with steeper $\alpha_{\text {ox }}$ values (i.e. X-ray quiet compared to the UV) should be characterized by faster disc-winds. This is the result of the shape of the ionizing SED. When the X-ray luminosity increases compared to the UV, the innermost parts of the wind become fully ionized. As a result only the slower streamlines of the wind are observed, launched from further out. In this scenario, we may expect the outflow velocity to decrease with increasing X-ray luminosity, the opposite of what is observed in PDS 456.

Nonetheless the strong positive correlation in Fig. 3 does not preclude that MHD processes play an important role in providing the initial lift of the wind material off the disc (i.e., $v_{i}$ ). In this regard we have some evidence that confirms this scenario, where in the low-luminosity observation of PDS 456 in 2013 with Suzaku, a strong X-ray flare increased the luminosity by a factor of $\sim 4$ in just $\sim 50$ ks which was likely to be magnetically driven (M16). The disc-wind became stronger after the flare was observed, supporting a scenario whereby MHD processes contributed at least to the initial ejection of the material off the disc, only for it to be then accelerated by the intense radiation pressure. Other AGN that accrete near the Eddington limit, e.g. in luminous quasars and Narrow Line Seyfert 1 Galaxies such as: APM 08279+5255 (Chartas, Brandt \& Gallagher 2003), PG1211+143 (Pounds et al.2003), IRAS F11119+3257 (Tombesi et al. 2015), 1H 0707-495 (Hagino et al.2016), IRAS 13224-3809 (Parker et al. 2017), are also likely to be promising candidates for radiatively driven winds. However the contribution of MHD winds are likely to be more important at the lower $L / L_{\mathrm{Edd}}$ ratio end of the population, where radiation driving would be difficult without large force multiplier factors.

\section{CONCLUSIONS}

We analysed all twelve X-ray observations of the fast disc-wind in PDS 456 from 2001 to 2014, observed through its iron K absorption profile. For the first time we find a positive correlation between the wind velocity and the intrinsic hard X-ray luminosity. This provides evidence that the accretion disc-wind in PDS 456 is most likely to be radiatively driven, with higher luminosities helping to accelerate the wind to higher terminal velocities.

\section{ACKNOWLEDGEMENTS}

We thank the anonymous referee for their helpful report. GM and VB acknowledge support from the Italian Space Agency (ASI INAF NuSTAR I/037/12/0). JR, DML and AL acknowledge the support of STFC. EN is supported by EU's Marie SkłodowskaCurie grant no. 664931. FT acknowledges support by the Programma per Giovani Ricercatori - 2014 "Rita Levi Montalcini”.

\section{REFERENCES}

Akritas M. G., Bershady M. A., 1996, ApJ, 470, 706

Behar E., Kaspi S., Reeves J., Turner T. J., Mushotzky R., O’Brien P. T., 2010, ApJ, 712, 26

Chartas G., Brandt W. N., Gallagher S. C., 2003, ApJ, 595, 85

Chartas G., Brandt W. N., Gallagher S. C., Garmire G. P., 2002, ApJ, 579, 169

Di Matteo T., Springel V., Hernquist L., 2005, Nature, 433, 604

Fiore F. et al., 2017, A\&A, 601, A143

Fukumura K., Kazanas D., Contopoulos I., Behar E., 2010, ApJ, 715,636

Fukumura K., Kazanas D., Shrader C., Behar E., Tombesi F., Contopoulos I., 2017, Nature Astronomy, 1, 0062

Gofford J. et al., 2014, ApJ, 784, 77

Gofford J., Reeves J. N., McLaughlin D. E., Braito V., Turner T. J.,

Tombesi F., Cappi M., 2015, MNRAS, 451, 4169

Gofford J., Reeves J. N., Tombesi F., Braito V., Turner T. J., Miller

L., Cappi M., 2013, MNRAS, 430, 60

Hagino K., Odaka H., Done C., Gandhi P., Watanabe S., Sako M., Takahashi T., 2015, MNRAS, 446, 663

Hagino K., Odaka H., Done C., Tomaru R., Watanabe S., Takahashi T., 2016, MNRAS, 461, 3954

Hopkins P. F., Elvis M., 2010, MNRAS, 401, 7

Kazanas D., Fukumura K., Behar E., Contopoulos I., Shrader C., 2012, The Astronomical Review, 7, 92

King A., 2003, ApJL, 596, L27

King A. R., Pounds K. A., 2003, MNRAS, 345, 657

Matzeu G. A., Reeves J. N., Nardini E., Braito V., Costa M. T., Tombesi F., Gofford J., 2016, MNRAS, 458, 1311

Matzeu G. A., Reeves J. N., Nardini E., Braito V., Turner T. J., Costa M. T., 2017, MNRAS, 465, 2804

Nardini E. et al., 2015, Science, 347, 860

Nomura M., Ohsuga K., 2017, MNRAS, 465, 2873

O’Brien P. T., Reeves J. N., Simpson C., Ward M. J., 2005, MNRAS, 360, L25

Ohsuga K., Mineshige S., Mori M., Kato Y., 2009, PASJ, 61, L7

Parker M. L. et al., 2017, Nature, 543, 83

Pounds K. A., Reeves J. N., King A. R., Page K. L., O'Brien P. T.,

Turner M. J. L., 2003, MNRAS, 345, 705

Proga D., Kallman T. R., 2004, ApJ, 616, 688

Proga D., Stone J. M., Kallman T. R., 2000, ApJ, 543, 686

Reeves J. N. et al., 2014, ApJ, 780, 45

Reeves J. N., Braito V., Nardini E., Behar E., O'Brien P. T., Tombesi F., Turner T. J., Costa M. T., 2016, ApJ, 824, 20

Reeves J. N. et al., 2009, ApJ, 701, 493

Reeves J. N., O'Brien P. T., Vaughan S., Law-Green D., Ward M., Simpson C., Pounds K. A., Edelson R., 2000, MNRAS, 312, L17

Reeves J. N., O’Brien P. T., Ward M. J., 2003, ApJ, 593, L65

Sim S. A., Miller L., Long K. S., Turner T. J., Reeves J. N., 2010, MNRAS, 404, 1369

Simpson C., Ward M., O’Brien P., Reeves J., 1999, MNRAS, 303, L23

Tombesi F., Cappi M., Reeves J. N., Palumbo G. G. C., Yaqoob T., Braito V., Dadina M., 2010, A\&A, 521, A57

Tombesi F., Meléndez M., Veilleux S., Reeves J. N., GonzálezAlfonso E., Reynolds C. S., 2015, Nature, 519, 436

Torres C. A. O., Quast G. R., Coziol R., Jablonski F., de la Reza R., Lépine J. R. D., Gregório-Hetem J., 1997, ApJL, 488, L19 